Journal for ImmunoTherapy of Cancer

\title{
Early tumor shrinkage identifies long- term disease control and survival in patients with lung cancer treated with atezolizumab
}

Ashley M Hopkins, Ganessan Kichenadasse, Chris S Karapetis, Andrew Rowland, Michael J Sorich

To cite: Hopkins AM, Kichenadasse G, Karapetis CS, et al. Early tumor shrinkage identifies long-term disease control and survival in patients with lung cancer treated with atezolizumab. Journal for ImmunoTherapy of Cancer 2020;8:e000500. doi:10.1136/ jitc-2019-000500

- Additional material is published online only. To view please visit the journal online (http://dx.doi.org/10.1136/jitc2019-000500).

AR and MJS contributed equally.

Accepted 09 April 2020

Check for updates

(C) Author(s) (or their employer(s)) 2020. Re-use permitted under CC BY-NC. No commercial re-use. See rights and permissions. Published by BMJ.

College of Medicine and Public Health, Flinders University,

Adelaide, South Australia,

Australia

Correspondence to

Dr Ashley M Hopkins;

ashley.hopkins@flinders.edu.au

\section{ABSTRACT}

Background Preliminary evidence indicates that early tumor shrinkage (ETS) following immune checkpoint inhibitor (ICI) initiation may be associated with survival outcomes in patients with advanced melanoma. ETS has not been explored as a biomarker of survival outcomes or patient-reported outcomes in patients with advanced nonsmall cell lung cancer (NSCLC) treated with ICls.

Methods The study pooled data from patients with NSCLC in the randomized trials OAK and POPLAR (atezolizumab vs docetaxel; $n=1464$ ), and single-arm atezolizumab trials BIRCH and FIR $(n=797)$. The association between ETS ( $\geq 10 \%$ decrease in pretreatment sum-of-longest diameters of target-lesions at 6 weeks) and overall survival (OS), progression-free survival (PFS), time to deterioration (TDD) in health-related quality-of-life (HRQoL) and physical function (PF) was assessed using Cox proportional hazard analysis.

Results ETS occurred in $20 \%$ of atezolizumab-treated patients with NSCLC within OAK and POPLAR and was associated with highly favorable OS (HR $0.33, p<0.001$ ), PFS (HR 0.31, $p<0.001$ ), TDD in HRQoL (HR 0.73, $p=0.01$ ) and $P F(H R 0.52, p<0.001)$. The results were replicated in the BIRCH and FIR data. Atezolizumab-treated patients achieving ETS had markedly improved OS compared with docetaxel-treated patients achieving ETS (24-month OS $55 \%$ vs $32 \%$ ); PFS was also markedly improved (24-month PFS $31 \%$ vs $4 \%$ ). In contrast, for patients not achieving ETS, atezolizumab-treatment was associated with more modest OS (24-month OS $23 \%$ vs $20 \%$ ) and PFS (24-month PFS $3 \%$ vs $1 \%$ ) improvement compared with docetaxel. Overall, the effect size for ETS within the atezolizumab-treated patients was significantly greater than that in the docetaxel-treated patients (P(interaction) $=0.002$ for $0 S$ and $P($ interaction $)<0.001$ for PFS).

Conclusions ETS is an easily measurable biomarker, predictive of highly favorable survival and patient-reported outcomes with atezolizumab treatment for advanced NSCLC. Further, ETS identifies patients with significantly greater treatment benefit for ICI therapy.

\section{INTRODUCTION}

Immune checkpoint inhibitors (ICIs) have demonstrated significant improvements in survival outcomes compared with conventional therapies across a range of cancers, including melanoma, non-small cell lung cancer (NSCLC) and renal cell carcinoma. However, substantial heterogeneity in survival outcomes between patients treated with ICIs have been observed.

Despite extensive research no pretreatment or early on therapy biomarker (including programmed death-ligand 1 (PD-L1) expression) has demonstrated a consistent ability to differentiate patients who will achieve long-term survival or treatment-benefit from ICI therapy. ${ }^{1-6}$ Minimal research has been conducted to identify biomarkers of quality of life changes from ICI therapies. Recent evidence highlights that atezolizumab-treated patients with NSCLC achieve survival benefit across all the best-response subgroups, ${ }^{7}$ a tumor growth model based on the first 40 weeks of tumor kinetic data can predict overall survival (OS), ${ }^{8}$ and a $10 \%$ reduction in tumor size early after ICI initiation in patients with metastatic melanoma is associated with improved OS. ${ }^{9}$ This preliminary evidence indicates that early tumor shrinkage (ETS) may be prognostic of survival outcomes in patients with advanced cancer treated with ICIs. However, to date, ETS has not been explored either as a biomarker of survival outcomes or patient-reported outcomes for patients with advanced NSCLC treated with ICIs.

ETS following treatment initiation has been indicated as an easy to measure and simple to determine clinical biomarker that is prognostic of survival outcomes across several cancers and therapies. ${ }^{10-15}$ Of interest is that ETS is available more rapidly than conventionally used best response criteria according to Response Evaluation Criteria in Solid Tumors (RECIST); and RECIST defines 
responders by a $30 \%$ reduction in the sum-of-longest diameters (SLD) of target-lesions, yet prognostic information may be identifiable across the range. ${ }^{10-15}$ Further, ETS may be a potential biomarker for go, no-go decisions in clinical trials. ${ }^{9}$ Regarding ICIs, the appropriateness of RECIST has been questioned considering observations of pseudoprogression and hyper-progression, and it is unclear if these concerns apply to ETS.

The objectives of this study were to identify early changes in tumor size associated with improved survival and patient-reported outcomes in patients with advanced NSCLC treated with the PD-L1 inhibitor atezolizumab.

\section{METHODS \\ Patients}

This study was a retrospective pooled analysis of individual participant data from the per-protocol populations of the clinical trials OAK $^{1617}$ (NCT02008227, July 7, 2016 data cut-off), POPLAR ${ }^{18}$ (NCT01903993, May 8, 2015 data cut-off), BIRCH $^{4}$ (NCT02031458, May 28, 2015 data cut-off), and FIR ${ }^{5}$ (NCT01846416, Jan 7, 2015 data cut-off). Secondary analysis of anonymized clinical trial data was deemed negligible risk research by the Southern Adelaide Local Health Network, Office for Research and Ethics and was exempt from review. Data were accessed according to Roche's policy and process for clinical study data sharing. ${ }^{19}$

OAK and POPLAR were randomized trials of atezolizumab $1200 \mathrm{mg}$ intravenous every 3 weeks versus docetaxel $75 \mathrm{mg} / \mathrm{m}^{2}$ intravenous every 3 weeks for patients with advanced NSCLC who have failed platinumcontaining therapy. ${ }^{16}$ Primary analyses within this study were pooled analyses of OAK and POPLAR which due to randomized design allow the comparison of assessed associations between treatments (atezolizumab vs docetaxel); unless otherwise stated results refer to OAK and POPLAR.

BIRCH and FIR were single-arm studies of atezolizumab $1200 \mathrm{mg}$ intravenous every 3 weeks in PD-L1-positive patients with advanced NSCLC ${ }^{45}$ Pooled analyses of BIRCH and FIR were used to demonstrate consistency of identified associations within an external cohort of patients treated with atezolizumab.

\section{Predictor and outcome definitions}

The coprimary outcomes assessed were OS and PFS. Secondary outcomes assessed were time to deterioration (TDD) in health-related quality-of-life (HRQoL) and physical function (PF).

Best overall response (BOR) and PFS was investigator assessed for POPLAR and OAK and defined by RECIST V.1.1. ${ }^{18}$ An independent review facility assessed BOR and PFS via RECIST V.1.1 for BIRCH, ${ }^{4}$ and they were investigator assessed per modified RECIST for FIR. ${ }^{5}$

For all trials, patient-reported outcomes (HRQoL and $\mathrm{PF}$ ) were assessed via the European Organisation for Research and Treatment of Cancer (EORTC) QLQC30 and QLQ-LC13. Study participants completed the
QLQ-C30 and QLQ-LC13 at baseline and day 1 of each cycle. PF was measured using a 4-point scale, ranging from 'not at all' to 'very much'. The global health status scale was used to measure HRQoL, and comprised of two questions on a 7-point scale reflecting the patients' overall health and QoL. ${ }^{17}$ Responses were scored according to the EORTC scoring manual third edition (standardizing raw responses to a $0-100$ point range).$^{20} \mathrm{~A} 10$-point change in a score is perceived by patients as clinically meaningful. ${ }^{21}$ Time to confirmed symptomatic deterioration was therefore defined as the time from baseline to the first time the score showed a $\geq 10$-point increase, which was confirmed over at least two consecutive cycles or accompanied by death within 3 weeks of the last assessment.

Tumor assessment and the calculation of SLD of target-lesions (per RECIST) was carried out at 6-week intervals within OAK, ${ }^{16}$ POPLAR, ${ }^{18}$ BIRCH,${ }^{4}$ and FIR. ${ }^{5}$ Early changes in tumor size $(\Delta \mathrm{TS})$ were defined by the percentage change in pretreatment (screening) SLD at the 6 -week visit.

\section{Statistical analysis}

All analyses were based on the per-protocol populations (ie, assessment of participants based on actual treatment received).

Univariable and multivariable associations between $\triangle \mathrm{TS}$ and OS, PFS, TDD in HRQoL and PF were modeled using Cox proportional hazards regression and reported as HRs with 95\% CIs and concordance statistics (c). All multivariable analyses were adjusted for pretreatment Eastern Cooperative Oncology Group performance status (ECOG PS), age, sex, race, smoking status, histology, count of prior treatments, PD-L1 expression, serum lactate dehydrogenase (LDH) levels and the presence of liver, lung or brain lesions-adjusted analyses were conducted to assess the independence of associations from other factors. All statistical regression analyses were stratified by study. Potential non-linear associations between $\Delta \mathrm{TS}$ and $\mathrm{OS} / \mathrm{PFS}$ were explored using restricted cubic splines, with model fit assessed via the Akaike information criterion (AIC). Analyses were conducted with a focus on facilitating clinical use and interpretability. $\Delta \mathrm{TS}$ was categorized based on model fit (assessed via the AIC), observed non-linear effects, and interpretable reference cut-points. A sensitivity recursive partitioning analysis was conducted to identify within atezolizumab-treated patients who experienced a decrease in pretreatment SLD at the 6-week visit, the $\Delta$ TS cut-off associated with robust prediction of patients who will achieve improved OS and PFS.

The distribution of pretreatment characteristics and BOR for patients with advanced NSCLC with and without ETS (defined by a $10 \%$ or greater decrease in the pretreatment SLD at the 6-week visit) following the initiation of atezolizumab or docetaxel were explored using the Fisher test for categorical data and Wilcoxon test for continuous data. Explored pretreatment characteristics included age, sex, race, smoking status, histology, ECOG PS, count of 


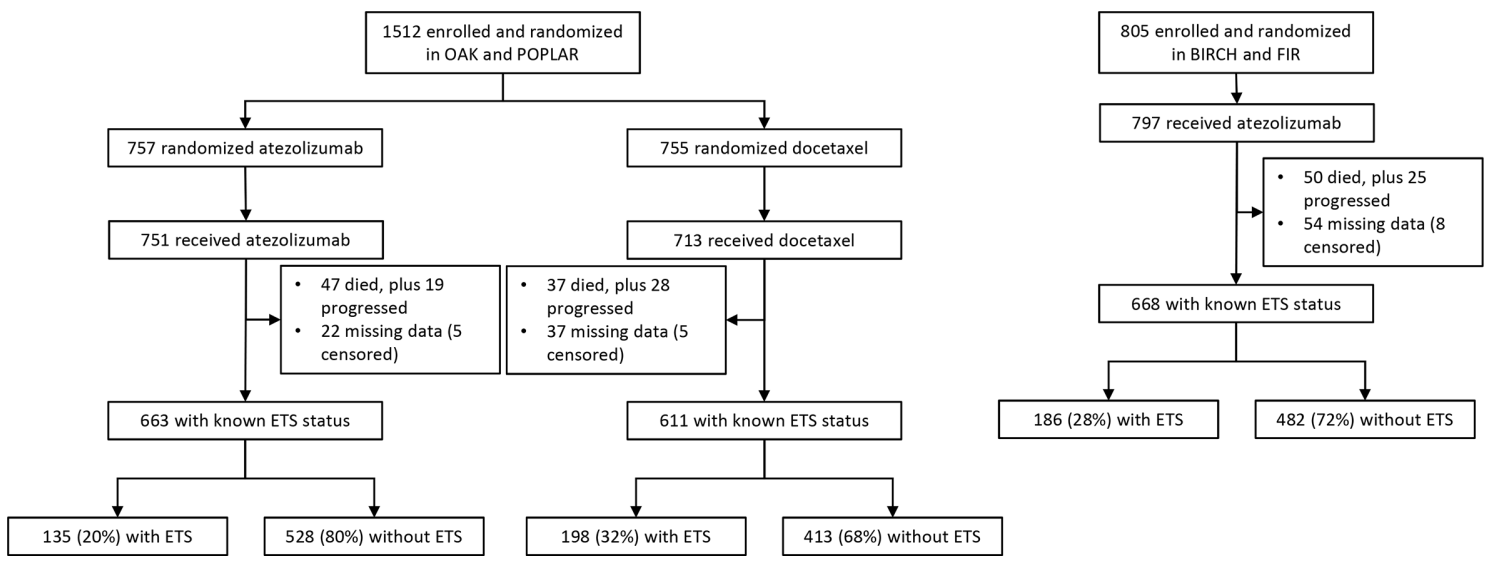

Figure 1 Flow diagram of study populations from OAK and POPLAR, plus BIRCH and FIR.ETS, early tumor shrinkage

prior treatments, time since diagnosis, stage, count of tumor sites, presence of liver/lung/brain lesions, LDH levels, PD-L1 expression, EGFR/KRAS mutation status, and EMLA-ALK rearrangement.

Exploratory analysis of the prognostic effect of ETS on OS/PFS by pretreatment characteristic subgroups was modeled by Cox proportional hazards regression and presented in a forest plot (online supplementary figures S3 and S4). Heterogeneity of treatment effect on OS/PFS according to patients with and without ETS was assessed using a treatment-by-biomarker interaction term in a Cox proportional regression model. A sensitivity analysis of the association between ETS status at 12 weeks with OS and PFS was conducted.

Kaplan-Meier analysis was used for plotting and estimating probabilities of OS, PFS, TDD, HRQoL and PF. All $p$ values $<0.05$ were considered statistically significant. Statistical analyses were performed using the $\mathrm{R}$ statistical environment (V.3.4, survival and rms packages).

\section{RESULTS}

Across all four clinical trials, 1942 patients with advanced NSCLC with treatment, outcome and $\Delta \mathrm{TS}$ data at 6 weeks were available. Figure 1 provides a summary of the analysable population from OAK/POPLAR, and BIRCH/ FIR. Median follow-up was 19 months (95\% CI: 19 to 20) within the cohort of 1274 from OAK and POPLAR.

\section{Identifying ETS cut-off}

On univariable and multivariable analysis, $\Delta$ TS (175 to 30 vs 30 to 10 vs 10 to -10 vs -10 to -30 vs -30 to -100 ) was significantly associated with OS $(\mathrm{p}<0.001)$, PFS $(p<0.001)$, TDD in HRQoL $(p<0.01)$ and PF $(p<0.001)$ in patients treated with atezolizumab from OAK and POPLAR (table 1). Online supplementary figure S1 presents Kaplan-Meier estimates of OS, PFS, TDD in HRQoL and $\mathrm{PF}$ for atezolizumab-treated patients by $\triangle \mathrm{TS}$ from OAK and POPLAR. Increases in $\triangle \mathrm{TS}$ were associated with worse outcomes in patients treated with atezolizumab (compared with $\Delta \mathrm{TS}$ of 10 to -10 ), with outcomes worst with increases greater than $30 \%$. Conversely, decreases in $\Delta \mathrm{TS}$ were associated with improved outcomes, with best outcomes achieved with decreases greater than $30 \%$. As a biomarker to identify patients likely to achieve improved survival and patient-reported outcomes from atezolizumab, a decrease cut-off of $10 \%$ or greater in the pretreatment SLD at the 6-week visit appears appropriate-consistent with Wang et $a .^{9}{ }^{9}$

Online supplementary figure S2 presents the continuous relationship ( $\log$ relative hazard $\pm 95 \%$ CI) between $\triangle \mathrm{TS}$ with OS and PFS for patients treated with atezolizumab and docetaxel from OAK/ POPLAR. The decrease cut-off of $10 \%$ or greater is observed as a key inflection point for patients who will achieve varying OS and PFS outcomes from atezolizumab therapy. Recursive partitioning analysis identified that for atezolizumab-treated patients from OAK/ POPLAR who experienced tumor shrinkage, the $\triangle T$ TS cut-off associated with robust prediction of improved OS and PFS was decreases greater than $10.5 \%$ and $10.3 \%$, respectively-further supporting a $10 \%$ or greater cut-off.

For atezolizumab-treated patients from BIRCH and FIR, exploratory analysis indicated an association between $\Delta$ TS and OS $(p<0.001)$, PFS $(p<0.001)$, TDD in HRQoL $(\mathrm{p}<0.001)$ and $\mathrm{PF}(\mathrm{p}<0.001$, online supplementary table S1). Consistent with the identified associations in the OAK and POPLAR cohort.

\section{ETS in atezolizumab-treated patients}

A significant univariable association between ETS (defined by a $10 \%$ or greater decrease in the pretreatment SLD at the 6-week visit) and improved OS (HR 0.33, $\mathrm{p}<0.001, c=0.58)$ and PFS (HR 0.31, $<<0.001, c=0.60)$ was observed in patients treated with atezolizumab from OAK and POPLAR (table 2). The association between ETS and improved OS (HR 0.32, $\mathrm{p}<0.001, \Delta c=0.02$ ) and PFS (HR $0.31, \mathrm{p}<0.001, \Delta c=0.05)$ was maintained on adjusted analysis (table 2)-demonstrating an independence of ETS from other known prognostic factors. Table 2 presents 24-month probabilities of OS, PFS, TDD in HRQoL and 
Table 1 Univariable and multivariable association between $\triangle T S$ and OS, PFS, TDD in HRQoL and PF for patients treated with atezolizumab from OAK and POPLAR

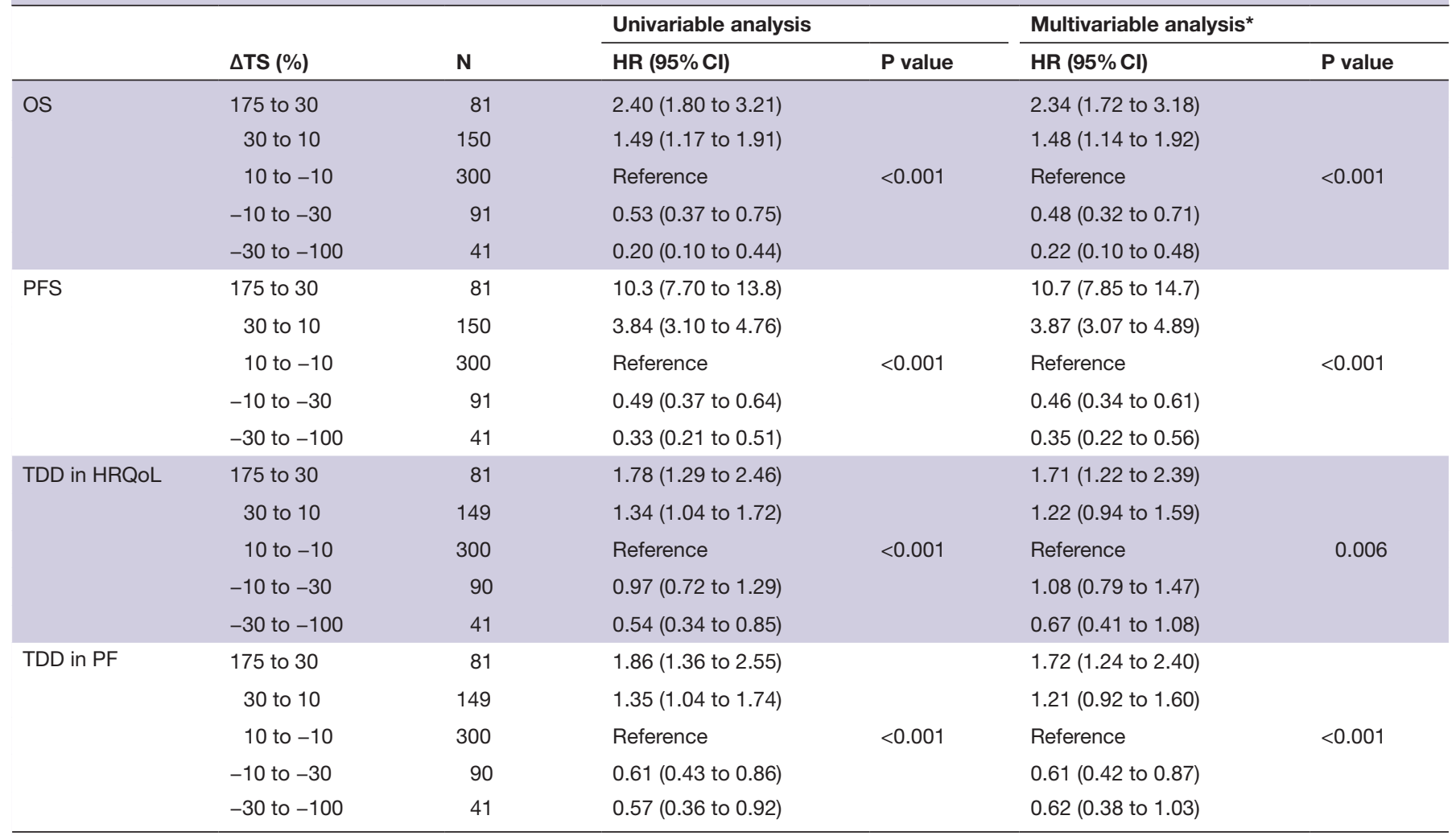

*Multivariable analysis adjusted for baseline ECOG PS, age, sex, race, smoking status, histology, count of prior treatments, PD-L1 expression, serum LDH levels and the presence of liver, lung or brain lesions.

ECOG PS, Eastern Cooperative Oncology Group performance status; HRQoL, health-related quality-of-life; LDH, lactate dehydrogenase; OS, overall survival; PD-L1, programmed death-ligand 1; PF, physical function; PFS, progression-free survival; TDD, time to deterioration; $\Delta T S$, early changes in tumor size; $\triangle T$, early changes in tumor size.

PF for atezolizumab-treated patients with and without ETS. Figure 2 presents Kaplan-Meier estimates of OS, PFS, TDD in HRQoL and PF for atezolizumab-treated patients with and without ETS.
For atezolizumab-treated patients from BIRCH and FIR, ETS was associated with improved OS $(p<0.001)$ and PFS $(p<0.001)$, aligning with the identified associations in the OAK and POPLAR cohort (online supplementary

Table 2 Univariable and multivariable association between ETS status (with and without) and OS, PFS, TDD in HRQLL and PF for patients treated with atezolizumab from OAK and POPLAR

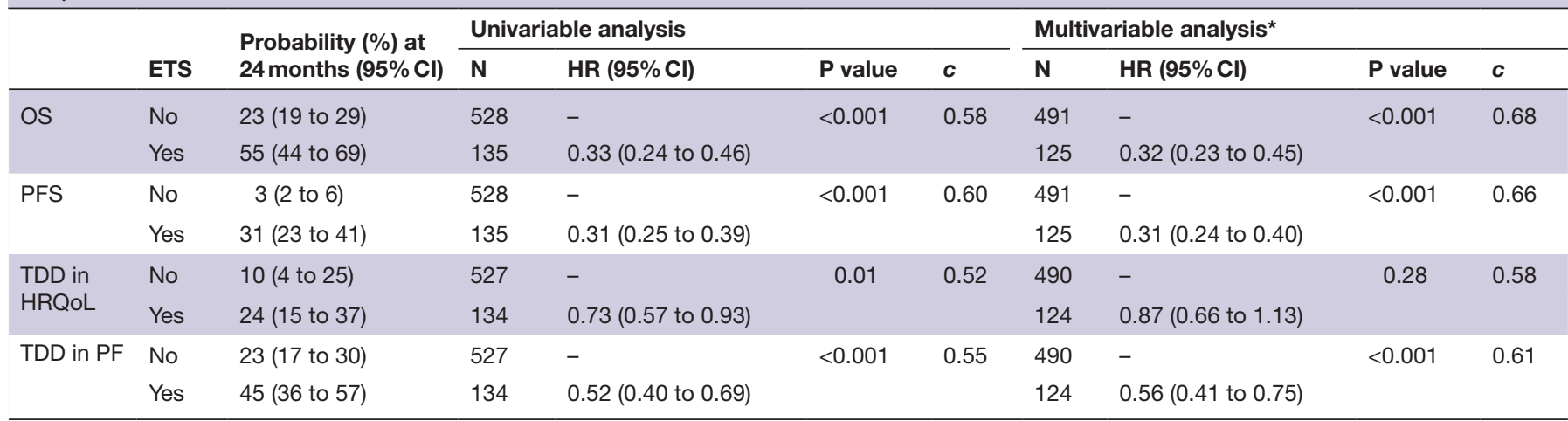

*Multivariable analysis adjusted for baseline ECOG PS, age, sex, race, smoking status, histology, count of prior treatments, PD-L1 expression, serum LDH levels and the presence of liver, lung or brain lesions.

ECOG PS, Eastern Cooperative Oncology Group performance status; HRQoL, health-related quality-of-life; LDH, lactate dehydrogenase; OS, overallsurvival; PD-L1, programmed death-ligand 1; PF, physical function; PFS, progression-free survival; TDD, time to deterioration. 

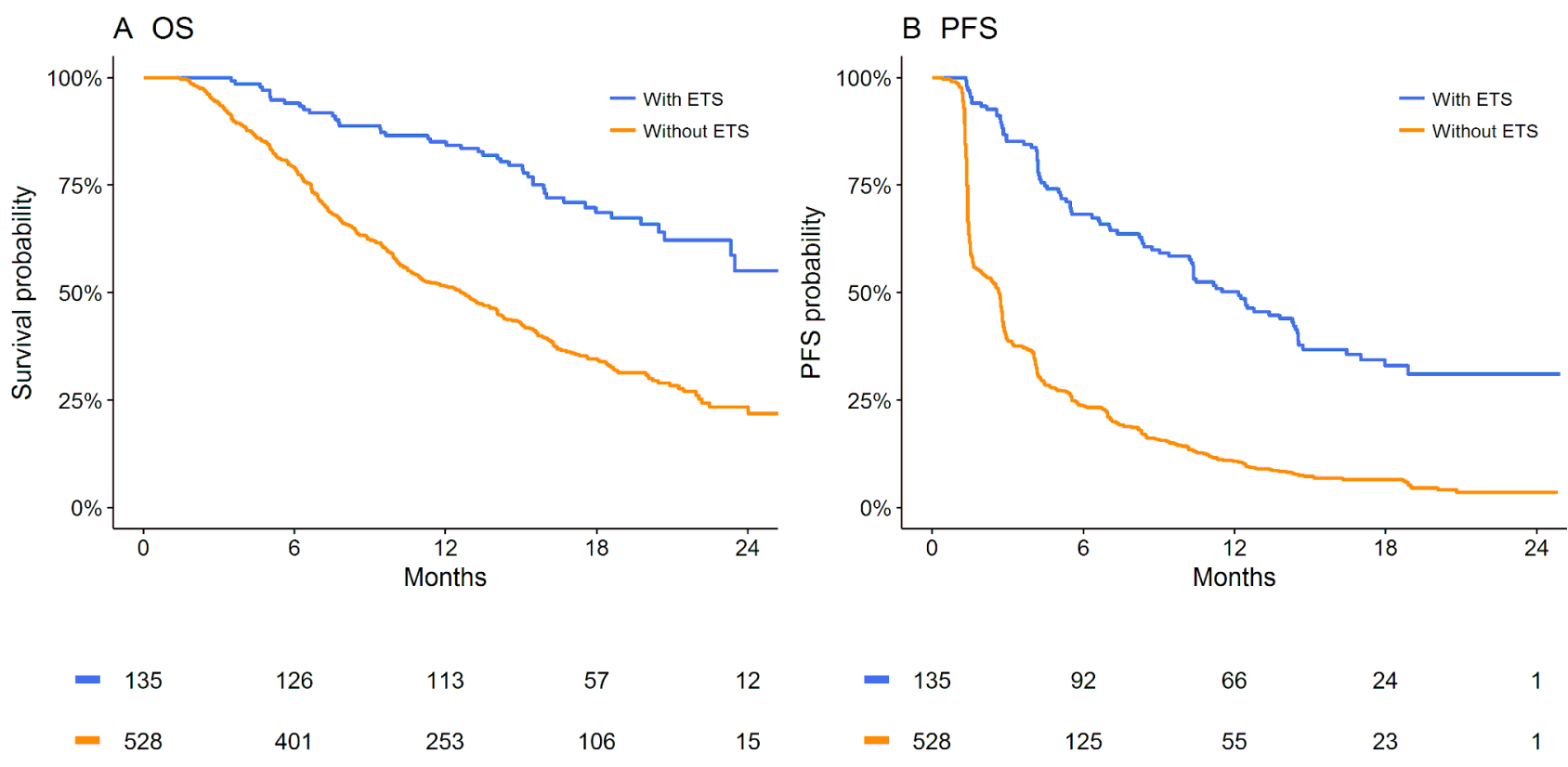

\section{TDD in HRQoL}
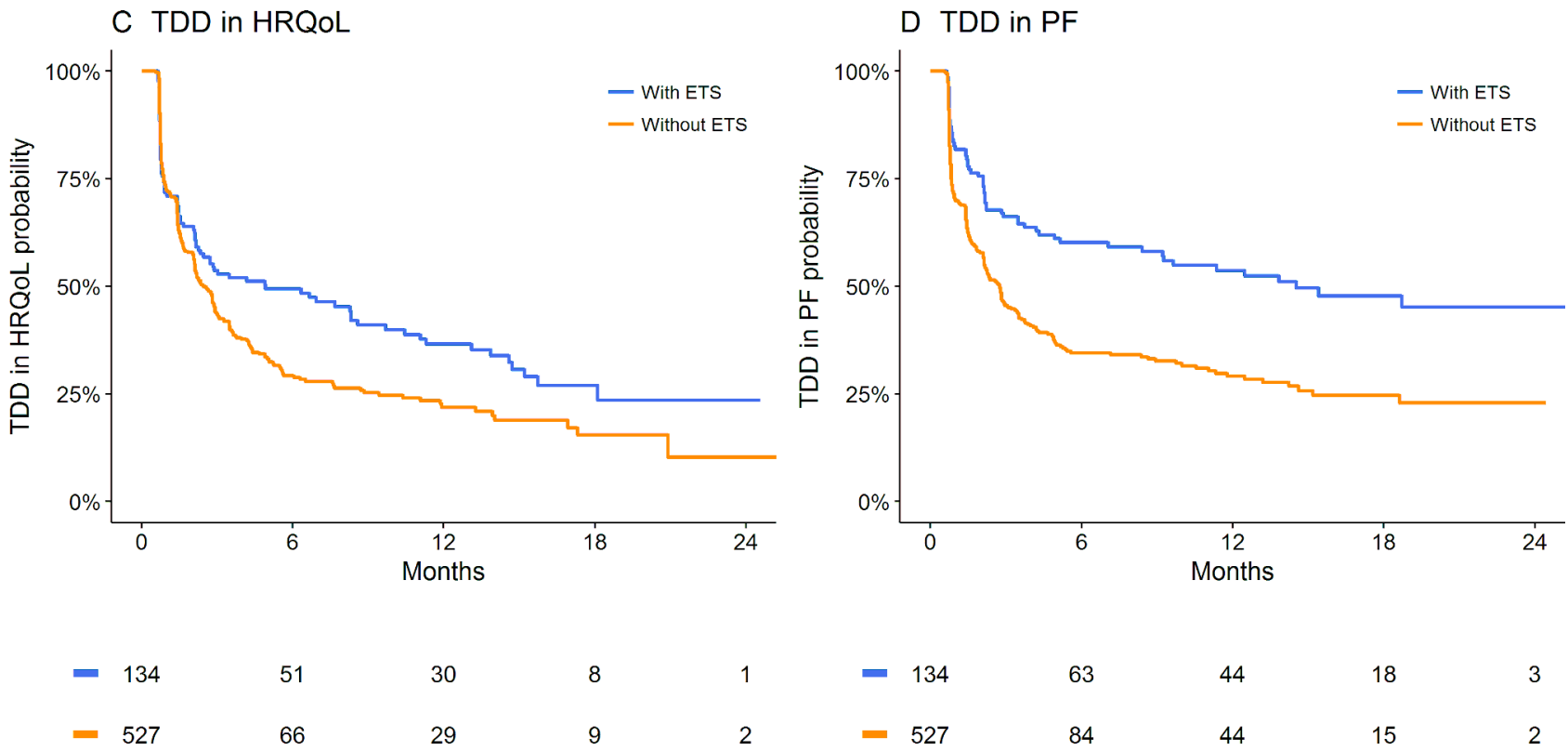

D TDD in PF

$\begin{array}{rrrrr}-134 & 51 & 30 & 8 & 1 \\ -527 & 66 & 29 & 9 & 2\end{array}$

- 527

84

44

15

2

Figure 2 Kaplan-Meier estimates of OS, PFS, TDD in HRQoL and PF by ETS status for atezolizumab-treated patients from OAK and POPLAR. ETS, early tumor shrinkage; HRQoL, health-related quality-of-life; OS, overall survival; PF, physical function; PFS, progression-free survival; TDD, time to deterioration.

table S2 and figure S5). ETS status at the 12-week visit was similarly associated with improved $\mathrm{OS}(\mathrm{p}<0.001)$ and PFS $(\mathrm{p}<0.001)$ within patients treated with atezolizumab from OAK/ POPLAR and BIRCH/ FIR (online supplementary tables S3 and S4).

Pretreatment characteristics of patients with advanced NSCLC with and without ETS following the initiation of atezolizumab are summarized in online supplementary tables S5 and S6. Within the OAK and POPLAR cohort of patients with advanced NSCLC treated with atezolizumab, the presence of ETS was associated with being a current smoker, lower tumor sites count, no lung tumors and PD-L1 expression $(\mathrm{p}<0.05)$. Within BIRCH and FIR, the presence of ETS was associated with a lower ECOG
PS, less prior treatments, no liver tumors and PD-L1 expression $(\mathrm{p}<0.05)$. Online supplementary tables $\mathrm{S} 7$ and $\mathrm{S} 8$ summarize the BOR of patients with advanced NSCLC according to ETS status following the initiation of atezolizumab within OAK/POPLAR and BIRCH/FIR, respectively.

\section{ETS in docetaxel-treated patients}

The presence of ETS was significantly associated with improved OS (univariable HR 0.61, $\mathrm{p}<0.001$; multivariable HR 0.65, $\mathrm{p}<0.001$ ) and PFS (univariable HR 0.61, $\mathrm{p}<0.001$; multivariable $\mathrm{HR} 0.64, \mathrm{p}<0.001)$ in patients treated with docetaxel (online supplementary table S9 and figure S6). However, the size of the effects within 
the docetaxel-treated patients was significantly smaller than that in the atezolizumab-treated patients $(\mathrm{P}$ (interaction $)=0.002$ for $\mathrm{OS}$ and $\mathrm{P}$ (interaction $)<0.001$ for PFS)-indicative that the magnitude of OS and PFS benefit from atezolizumab (compared with docetaxel) was greater in patients with ETS.

\section{Strength of ETS association with atezolizumab treatment compared to docetaxel}

For patients with ETS, the 24-month OS probability was $55 \%$ (95\% CI; $44 \%$ to $69 \%)$ for atezolizumab-treated patients compared with $32 \%(23 \%-44 \%)$ for docetaxel, while 24-month PFS probability was $31 \%(23 \%-41 \%)$ vs $4 \%(2 \%-10 \%)$, respectively. For patients without ETS, the 24-month OS probability was $23 \%$ (95\% CI; $19 \%$ to

A OS - With ETS
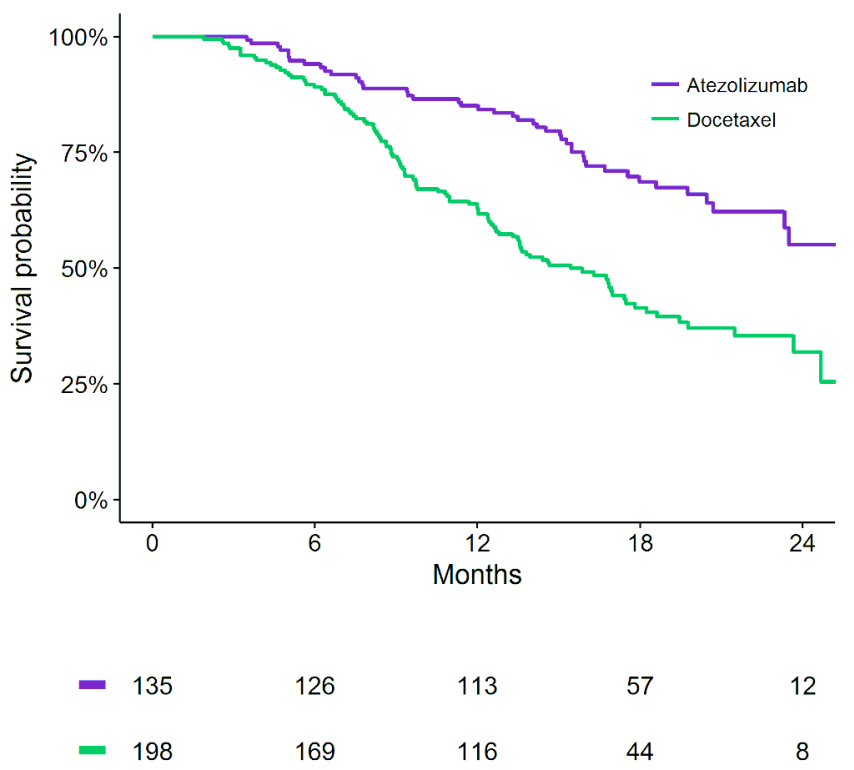

C PFS - With ETS

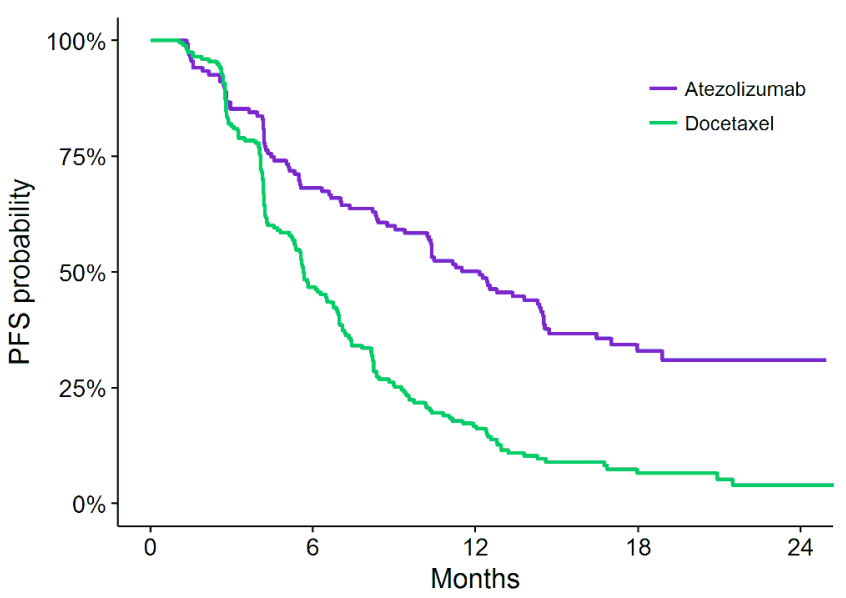

$\begin{array}{rrrrr}-135 & 92 & 66 & 24 & 1 \\ -198 & 87 & 29 & 8 & 1\end{array}$

$29 \%)$ for atezolizumab-treated patients compared with $20 \%(16 \%-26 \%)$ for docetaxel, while 24-month PFS probability was $3 \%(2 \%-6 \%)$ vs $1 \%(0 \%-5 \%)$, respectively. Figure 3 presents Kaplan-Meier estimates of the effect of atezolizumab (compared with docetaxel) on OS and PFS in patients with and without ETS.

\section{DISCUSSION}

For the first time, it has been demonstrated that in individuals treated with atezolizumab for advanced NSCLC, the presence of ETS is significantly associated with highly favorable survival outcomes and patient-reported outcomes. By evaluation of ETS in both the atezolizumab
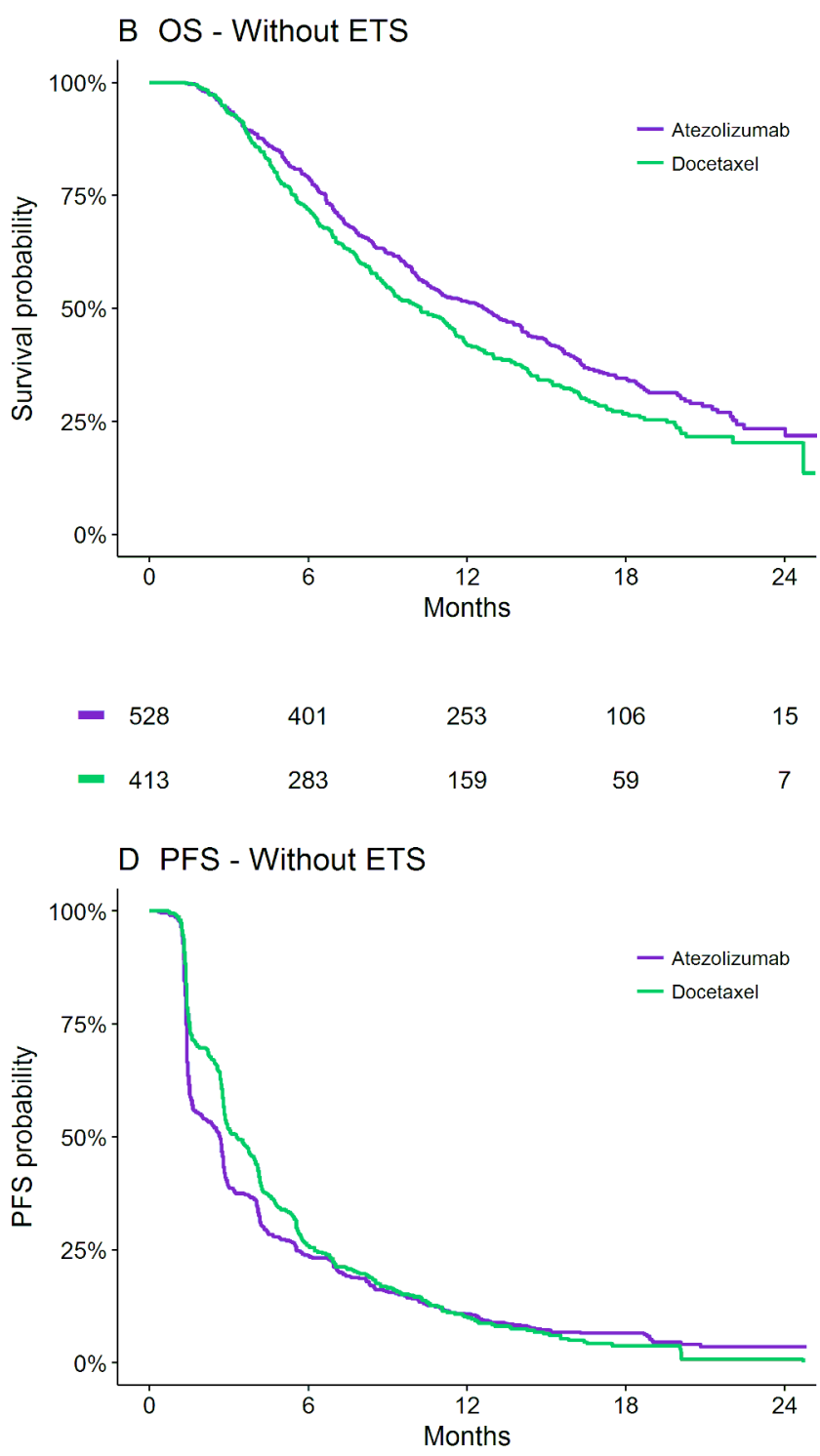

$\begin{array}{rrrrr}528 & 125 & 55 & 23 & 1 \\ -\quad 413 & 99 & 34 & 8 & 1\end{array}$

Figure 3 Kaplan-Meier estimates of OS and PFS for atezolizumab versus docetaxel-treated patients from OAK and POPLAR with (A, C) and without (B, D) ETS. ETS, early tumor shrinkage; OS, overall survival; PFS, progression-free survival. 
and docetaxel arms of the randomized OAK and POPLAR trials, it was demonstrated that the magnitude of OS and PFS benefit of atezolizumab was significantly greater in patients with ETS-indicative that ETS may be a biomarker of those likely to achieve amplified disease control and survival benefit from ICI therapy. This is the debut demonstration that ETS identifies greater benefit (cf. favorable prognosis) from ICI use.

The strength of ETS as a biomarker is that it is easily measurable in routine clinical practice and occurs early enough to impact clinical decisions for most patients. The strengths of this study are the large sample size, high-quality data collection, randomized allocation of treatment, and confirmation of the ETS association with survival outcomes for atezolizumab-treated patients in a second cohort (BIRCH and FIR studies). To the best of the authors knowledge, this is the first study to assess the association between ETS and survival outcomes in an ICI-treated cohort for NSCLC, and the first to identify heterogeneity of ICI treatment benefit (ie, treatment-bycovariate interaction) by ETS status. Further, it is the first study to assess the association between ETS and patientreported outcomes following the initiation of cancer treatments in the advanced setting.

This study demonstrates ETS (as defined $\geq 10 \%$ decrease in the pretreatment SLD at 6 weeks) to be a prognostic biomarker associated with improved OS, PFS, TDD in HRQoL and PF for patients with NSCLC using atezolizumab. Further, multivariable analysis demonstrated ETS to be independently associated with improved OS and PFS. These results are in accordance with Wang et a $\hat{l}^{p}$ who demonstrated that decreases of $10 \%$ or greater in tumor size at 12 and 18 weeks are strongly associated with improved survival in patients with metastatic melanoma treated with immunotherapies (pembrolizumab or ipilimumab). Importantly, the study herein had tumor size data available at an earlier 6 weeks, beneficial in an oncology environment where early switching of unsuccessful therapies is crucial. Further, the study included data from patients with NSCLC randomly allocated to docetaxel within OAK and POPLAR. ${ }^{16}{ }^{18}$ Such data allowed between treatment comparisons, identifying that while ETS is also prognostic of OS and PFS in patients with NSCLC using docetaxel, interestingly, the strength of ETS as a biomarker of outcomes is much greater for atezolizumab. For example, the 24-month OS probability with docetaxel treatment was 32\% for patients achieving ETS and 20\% for patients not achieving ETS. In contrast, the differences in 24-month OS probability with atezolizumab treatment differed substantially more $(55 \%$ for patients achieving ETS and 23\% for patients not achieving ETS), suggesting ETS is a particularly effective early biomarker for immunotherapies.

While no large benefit in 24-month OS and PFS probability was apparent in atezolizumab-treated patients compared with docetaxel-treated patients who did not achieve ETS, this must be interpreted cautiously as ETS is a discriminator occurring after baseline which confounds randomized arm assessments (ie, atezolizumab vs docetaxel). Hence, direct Cox proportional hazard assessments for these subgroups were not conducted. A primary finding of this study is that the magnitude of atezolizumab benefit on OS and PFS is significantly greater in patients with ETS, which indicates ETS may be a biomarker reflective of a cohort likely to achieve amplified chances of long-term disease control and survival from ICIs as compared with conventional therapies-this is not an intuitive a priori when considering potential pseudoprogression. Conversely, the study observed considerably worse outcomes (online supplementary figure S1) for atezolizumab-treated patients with increases in $\Delta \mathrm{TS}$ above $30 \%$ as compared with increases between $10 \%$ and $30 \%$-such findings are consistent with reports of the potential for ICIs to induce hyper-progression.

Limitations of this study are the restricted range of ICIs and settings evaluated. Variable subsequent therapies between patients following study completion may have also influenced identified associations between ETS and OS, although this is unlikely to have markedly impacted as the identified associations were generally consistent with that identified for PFS. As there is currently no gold standard to assess $\Delta \mathrm{TS}$, future research will need to evaluate the robustness of identified associations in the context of heterogeneity between centers and radiological experts. To minimize this potential confounder no $\Delta \mathrm{TS}$ was defined as within $\pm 10 \%$ of the pretreatment SLD at 6 weeks. ${ }^{9}$ As improved methods for measuring tumors and microtumors are developed this cut-off may need re-evaluation, as well as appreciation of current nontarget lesion definitions.

The key differences between OAK/ POPLAR and BIRCH/ FIR are that BIRCH and FIR include only PD-L1positive patients and these trials were not limited to patients whose disease had progressed on prior platinumcontaining therapy. Despite these differences the identified associations between ETS and outcomes were largely consistent between the cohorts. However, variations in pretreatment characteristics associated with achieving ETS were observed, likely driven by trial differences. Key pretreatment characteristics associated with achieving ETS appeared to be tumor burden metrics, line of therapy and PD-L1 expression.

Future directions of research include evaluation of ETS as a biomarker for other ICIs used in advanced NSCLC treatment (durvalumab, nivolumab, pembrolizumab), for first-line use of ICIs, and ICIs used in combination therapies. If the amplified strength of ETS as a disease control and survival biomarker for ICIs is confirmed (as compared with its modest performance for chemotherapies), consideration may be given to evaluating ETS as an ICI trial endpoint to accelerate clinical and early evidence development. Moreover, ETS may be considered as an early read-out for pay for performance strategies, or for go, no-go decisions in prospective ICI trials, where, respectively, the absence of ETS signifies a marker to not pay, or to intensify ICI therapy through 
combination approaches/switching to an alternative ICI. Future research will also include greater evaluation of patient characteristics associated with substantially good or bad outcomes to ICIs with the occurrence of ETS.

\section{CONCLUSIONS}

ETS is an easily measurable, early biomarker associated with highly favorable survival and patient-reported outcomes with atezolizumab treatment for advanced NSCLC. Further, patients achieving ETS with atezolizumab treatment had markedly improved survival outcomes compared with patients achieving ETS with docetaxel treatment, indicating that ETS may be particularly predictive of patients with greatest benefit from ICI use.

Funding Undertaken with the financial support of Cancer Council South Australia's Beat Cancer Project on behalf of its donors and the State Government through the Department of Health (Grant ID: 1159924 and 1127220). A.R. is supported by a Beat Cancer Mid-Career Research Fellowship from Cancer Council SA. AMH is a researcher funded by a Postdoctoral Fellowship from the National Breast Cancer Foundation, Australia (PF-17-007).

Competing interests MJS and AR report investigator-initiated project grants from Pfizer, outside the scope of the submitted work. CSK reports advisory board roles with AstraZeneca, Merck Sharp \& Dohme, Bristol-Myers Squibb, and Roche, outside the submitted work.

Patient consent for publication Not required.

Ethics approval Secondary analysis of anonymized clinical trial data was deemed negligible risk research by the Southern Adelaide Local Health Network, Office for Research and Ethics and was exempt from review.

Provenance and peer review Not commissioned; externally peer reviewed.

Data availability statement Individual-participant data utilised in this study is available for request to access at clinicalstudydatarequest.com.

Open access This is an open access article distributed in accordance with the Creative Commons Attribution Non Commercial (CC BY-NC 4.0) license, which permits others to distribute, remix, adapt, build upon this work non-commercially, and license their derivative works on different terms, provided the original work is properly cited, appropriate credit is given, any changes made indicated, and the use is non-commercial. See http://creativecommons.org/licenses/by-nc/4.0/.

\section{REFERENCES}

1 Hopkins AM, Rowland A, Kichenadasse G, et al. Predicting response and toxicity to immune checkpoint inhibitors using routinely available blood and clinical markers. Br J Cancer 2017;117:913.

2 Prelaj A, Tay R, Ferrara R, et al. Predictive biomarkers of response for immune checkpoint inhibitors in non-small-cell lung cancer. Eur $J$ Cancer 2019;106:144-59.

3 Joseph RW, Elassaiss-Schaap J, Kefford R, et al. Baseline tumor size is an independent prognostic factor for overall survival in patients with melanoma treated with pembrolizumab. Clin Cancer Res 2018;24:4960-7.
4 Peters S, Gettinger S, Johnson ML, et al. Phase II trial of atezolizumab as first-line or subsequent therapy for patients with programmed death-ligand 1-selected advanced non-small-cell lung cancer (BIRCH). J Clin Oncol 2017;35:2781-9.

5 Spigel DR, Chaft JE, Gettinger S, et al. Fir: efficacy, safety, and biomarker analysis of a phase II open-label study of Atezolizumab in PD-L1-Selected patients with NSCLC. J Thorac Oncol 2018;13:1733-42.

6 Reck M, Rodríguez-Abreu D, Robinson AG, et al. Pembrolizumab versus chemotherapy for PD-L1-positive non-small-cell lung cancer. N Engl J Med 2016;375:1823-33.

7 von Pawel J, Bordoni R, Satouchi M, et al. Long-term survival in patients with advanced non-small-cell lung cancer treated with atezolizumab versus docetaxel: results from the randomised phase III OAK study. Eur J Cancer 2019;107:124-32.

8 Claret L, Jin JY, Ferté C, et al. A model of overall survival predicts treatment outcomes with Atezolizumab versus chemotherapy in nonsmall cell lung cancer based on early tumor kinetics. Clin Cancer Res 2018;24:3292-8.

9 Wang M, Chen C, Jemielita T, et al. Are tumor size changes predictive of survival for checkpoint blockade based immunotherapy in metastatic melanoma? J Immunother Cancer 2019;7:39.

10 Grünwald V, Dietrich M, Pond GR. Early tumor shrinkage is independently associated with improved overall survival among patients with metastatic renal cell carcinoma: a validation study using the COMPARZ cohort. World J Urol 2018;36:1423-9.

11 Cremolini C, Loupakis F, Antoniotti C, et al. Early tumor shrinkage and depth of response predict long-term outcome in metastatic colorectal cancer patients treated with first-line chemotherapy plus bevacizumab: results from phase III TRIBE trial by the Gruppo Oncologico del Nord Ovest. Ann Oncol 2015;26:1188-94.

12 Wei $\mathrm{M}, \mathrm{Ye} \mathrm{Q}$, Wang $\mathrm{X}$, et al. Early tumor shrinkage served as a prognostic factor for patients with stage III non-small cell lung cancer treated with concurrent chemoradiotherapy. Medicine 2018;97:e0632.

13 Petrelli F, Pietrantonio F, Cremolini C, et al. Early tumour shrinkage as a prognostic factor and surrogate end-point in colorectal cancer: a systematic review and pooled-analysis. Eur J Cancer 2015;51:800-7.

14 Sakamaki K, Kito Y, Yamazaki K, et al. Exploration of time points and cut-off values for early tumour shrinkage to predict survival outcomes of patients with metastatic colorectal cancer treated with first-line chemotherapy using a biexponential model for change in tumour size. ESMO Open 2017;2:e000275.

15 Colloca GA, Venturino A, Guarneri D. Early tumor shrinkage after first-line medical treatment of metastatic colorectal cancer: a metaanalysis. Int J Clin Oncol 2019;24:231-40.

16 Rittmeyer A, Barlesi F, Waterkamp D, et al. Atezolizumab versus docetaxel in patients with previously treated non-small-cell lung cancer (OAK): a phase 3, open-label, multicentre randomised controlled trial. Lancet 2017;389:255-65.

17 Bordoni R, Ciardiello F, von Pawel J, et al. Patient-reported outcomes in OAK: a phase III study of atezolizumab versus docetaxel in advanced non-small-cell lung cancer. Clin Lung Cancer 2018;19:441-9.e4.

18 Fehrenbacher L, Spira A, Ballinger M, et al. Atezolizumab versus docetaxel for patients with previously treated non-small-cell lung cancer (POPLAR): a multicentre, open-label, phase 2 randomised controlled trial. Lancet 2016;387:1837-46.

19 Strom BL, Buyse M, Hughes J, et al. Data sharing, year 1--access to data from industry-sponsored clinical trials. N Engl J Med 2014;371:2052-4.

20 Fayers P, Aaronson NK, Bjordal K. EORTC QLQ-C30 scoring manual: European organisation for research and treatment of cancer, 2001.

21 Osoba D, Rodrigues G, Myles J, et al. Interpreting the significance of changes in health-related quality-of-life scores. J Clin Oncol 1998;16:139-44. 